Viviane Rohrig RABASSA ${ }^{1}$

Elizabeth SCHWEGLER ${ }^{1}$

Maikel Alan GOULART ${ }^{1}$

Mateus Silveira LOPES

Dustin André HOFFMANN ${ }^{1}$

Fernando Paixão LISBOA $^{1}$

Lúcio VENDRAMIN ${ }^{1}$

Victor Fernando Buttow ROLL ${ }^{2}$

Gonzalo J. DIAZ ${ }^{3}$

Francisco Augusto Burkert

DEL PINO ${ }^{1}$

Marcio Nunes CORRÊA ${ }^{1}$

Correspondência para:

Viviane Rohrig Rabassa, Universidade

Federal de Pelotas, Faculdade de

Veterinária - Departamento de Clínicas

Veterinária - Núcleo de Pesquisa

Ensino e Extensão em Pecuária

(NUPEEC) - Campus Universitário -

96010900 - Pelotas/RS - Brasil

vivianer@ufpel.edu.br;

nupeec@ufpel.edu.br;

www.ufpel.edu.br/nupeec

Recebido para publicação: 13/03/2009 Aprovado para publicação: 04/03/2010

\title{
Parâmetros metabólicos de ovelhas submetidas a dietas contendo aflatoxina e zearalenona com adição de glucomanano modificado
}

\author{
1- Núcleo de Pesquisa, Ensino e Extensão em Pecuária da Universidade \\ Federal de Pelotas, Pelotas-RS \\ 2- Departamento de Zootecnia da Universidade Federal de Pelotas, Pelotas- \\ RS \\ 3- Departamento de Ciencias en Salud Animal, Universidad Nacional de \\ Colombia, Bogotá
}

\section{Resumo}

As micotoxicoses são grandes causadoras de perdas produtivas em ruminantes, sendo aflatoxina (AFLA) e zearalenona (ZEA) as principais micotoxinas encontradas em alimentos conservados. Estas micotoxinas apresentam efeito sobre o metabolismo animal, através da ação anabólica de metabólitos da ZEA, bem como pelas lesões hepáticas causadas pela AFLA. O objetivo deste estudo foi determinar a influência do adsorvente glucomanano modificado sobre parâmetros metabólicos de ovelhas submetidas a dietas contendo AFLA e ZEA. Foram utilizadas 34 fêmeas divididas em 6 grupos (ZEA; ZEA + ADS; AFLA; AFLA + ADS; CONTROLE + ADS; CONTROLE), recebendo $1,0 \mathrm{mg} / \mathrm{kg}$ de ZEA, $1,5 \mathrm{mg} / \mathrm{kg}$ de AFLA e/ou $2 \mathrm{~kg} /$ ton de adsorvente. A ZEA diminuiu os níveis séricos de glicose, em relação ao CONTROLE $(\mathrm{p}<0,05)$, porém, o adsorvente não influenciou os níveis de glicose, não havendo diferença entre os grupos ZEA e ZEA + ADS. A ZEA aumentou os níveis de AST e GGT em relação ao grupo CONTROLE ( $\mathrm{p}<$ $0,05)$, sendo que os níveis de AST foram superiores no grupo ZEA $(\mathrm{p}<0,05)$, quando comparado ao grupo ZEA + ADS. Ainda, a aflatoxina causou uma redução nos níveis de albumina, em relação aos valores fisiológicos de ovinos. Assim, a partir destes resultados pode-se concluir que a ZEA causou alteraçóes metabólicas em ovinos, bem como o glucomanano modificado foi eficiente em reduzir a possível agressão hepática causada por esta micotoxina, demonstrada pela diminuição nos níveis de AST.

\section{Introdução}

Com o crescente aumento nos níveis produtivos de ruminantes, a adição de concentrados na dieta destes animais se tornou de grande importância. Porém, os componentes desta dieta concentrada e suas formas de conservação fornecem um ambiente adequado para o desenvolvimento de fungos, os quais produzem substâncias tóxicas denominadas micotoxinas, com efeitos prejudiciais aos índices produtivos de ruminantes ${ }^{1}$.
Palavras-chave: Aflatoxina. Zearalenona. Glucomanano modificado. Metabolismo. Ovinos.
A aflatoxina é uma das principais micotoxinas encontradas em alimentos conservados, sendo causadora de severas lesóes hepáticas, levando a perdas produtivas por diminuição no ganho de peso e eficiência alimentar ${ }^{2}$.

Dentre as micotoxinas que causam prejuízo à performance de ruminantes, também se destaca a zearalenona, produzida por várias espécies de Fusarium, devido à sua similaridade estrutural com o estradiol, causando alteraçôes reprodutivas ${ }^{3}$. Além 
disto, o $\alpha$-zearalenol, metabólico hidroxilado da zearalenona, pode ser convertido em zeranol por meio da hidrogenação no rúmen. Este último metabólito é um hormônio estrogênico, atuando como promotor de crescimento $^{4}$, podendo causar distúrbios metabólicos em ruminantes.

Uma importante forma de controle das micotoxicoses é a adição de substâncias adsorventes à dieta, evitando a absorção pelo epitélio intestinal. O efeito adsorvente do glucomanano modificado, obtido a partir da parede de leveduras, em especial a Saccharomyces cerevisae, tem sido amplamente estudado nas últimas décadas, em especial em aves e suínos ${ }^{5}$. Porém, ainda são poucos os resultados conhecidos em ruminantes, devido ao pequeno impacto econômico que as micotoxicoses apresentavam nas criaçôes extensivas. Entretanto, na intensificação da produção, torna-se um problema significativo na obtenção de um adequado desempenho produtivo de bovinos e ovinos confinados.

Vários estudos demonstraram as perdas produtivas geradas por estas micotoxicoses ${ }^{1,2}$, por meio da intoxicação clínica, porém não são conhecidos os efeitos subclínicos sobre o metabolismo de ruminantes, os quais geram perdas produtivas que muitas vezes não são percebidas pelo produtor.

O objetivo deste estudo foi determinar a influência da adição do adsorvente glucomanano modificado sobre parâmetros metabólicos de ovelhas submetidas a dietas contendo aflatoxina e zearalenona.

\section{Materiais e Métodos}

Este experimento foi realizado nas dependências do Hospital de Clínicas Veterinária da Universidade Federal de Pelotas (UFPel), Pelotas/RS, junto ao Centro de Experimentação em Ovinos (CEO) utilizando 34 fêmeas ovinas, obtidas do cruzamento entre as raças Corriedale e Texel, apresentando média de 1,5 anos de idade e 47,5 $\mathrm{kg}$ de peso corporal. As ovelhas utilizadas foram liberadas para uso em experimentos pela Comissão de Ética em
Experimentação Animal da UFPel, em 27 de março de 2007 (protocolo 010/2006).

As ovelhas foram mantidas confinadas, recebendo dieta à base de feno de alfafa $(90,5$ $\%$ matéria seca, $19,2 \%$ proteína bruta, 1,4 $\%$ cálcio, $0,2 \%$ fósforo), feno de tifton $(85,8$ $\%$ matéria seca, 5,8 \% proteína bruta, 4,8 $\%$ cinzas, $0,7 \%$ extrato etéreo, $25,7 \%$ fibra bruta) e concentrado (ingredientes: calcário calcítico, casca de soja moída, cloreto de sódio, farelo de arroz desengordurado, farelo de soja, milho moído, óleo vegetal, premix vitamínico mineral; níveis de garantia: $15 \%$ proteína bruta, $12 \%$ cinzas, $1,5 \%$ cálcio, $0,9 \%$ fósforo, $2 \%$ extrato etéreo, $13 \%$ fibra bruta), sendo este em quantidade equivalente a 1,5\% do peso vivo, numa relação volumoso/ concentrado de 65/35, apresentando níveis de micotoxinas compatíveis com os valores considerados aceitáveis pelo fabricante.

As fêmeas foram dividas entre seis grupos, sendo distribuídas uniformemente de acordo com a sua condição corporal (CC) e peso, estando a CC das ovelhas entre 3,0 e 4,0 (escala de 1,0 a 5,0 $)^{6}$. A tabela 1 apresenta a divisão de grupos utilizada no experimento, de acordo com a presença de aflatoxina, zearalenona e/ou adsorvente (Mycosorb ${ }^{\circledR}$ Alltech, Inc.) na dieta das fêmeas.

As micotoxinas foram produzidas pelo LAMIC (Laboratório de Análises Micotoxicológicas - UFSM), sendo obtidas pela metodologia de extração, clarificação e derivação totalmente automatizada, e análise por Cromatografia Líquida de Alta Eficiência (HPLC), tendo a zearalenona apresentado uma concentração de $7118,26 \mathrm{mg} / \mathrm{kg}$, enquanto que a concentração de aflatoxina foi de $224,75 \mathrm{mg} / \mathrm{kg}$. Ainda, a aflatoxina apresentou concentraçóes de 84,0 \%, 5,4\%, $9,1 \%$ e $1,5 \%$ de aflatoxinas B1, B2, G1 e $\mathrm{G} 2$, respectivamente.

As fêmeas foram adaptadas à dieta por um período de 21 dias antes da inclusão das micotoxinas e do adsorvente, sendo considerado o Dia 0 do experimento o dia do início do fornecimento destas substâncias.

A partir do Dia 0 foram realizadas coletas de sangue a cada 7 dias, por um período de 35 dias, totalizando 6 coletas. 
Tabela 1- Níveis de aflatoxina, zearalenona e glucomanano modificado (ADS) na dieta de cada grupo experimental

\begin{tabular}{lcccc}
\hline Grupo & $\mathbf{n}$ & Zearalenona $^{\mathbf{a}}$ & Aflatoxina $^{\mathbf{a}}$ & Adsorvente $^{\mathbf{a}}$ \\
\hline ZEA $^{1}$ & 6 & $1,0 \mathrm{mg} / \mathrm{kg}$ & - & - \\
AFLA $^{2}$ & 6 & - & $1,5 \mathrm{mg} / \mathrm{kg}$ & - \\
$Z \mathrm{ZEA}+\mathrm{ADS}^{3}$ & 6 & $1,0 \mathrm{mg} / \mathrm{kg}$ & - & $2 \mathrm{~kg} / \mathrm{ton}$ \\
$\mathrm{AFLA}+\mathrm{ADS}^{3}$ & 6 & - & $1,5 \mathrm{mg} / \mathrm{kg}$ & $2 \mathrm{~kg} / \mathrm{ton}$ \\
CONTROLE + ADS $^{3}$ & 5 & - & - & $2 \mathrm{~kg} / \mathrm{ton}$ \\
CONTROLE & 5 & - & - & - \\
\hline
\end{tabular}

a Nível de inclusão em relação à fração concentrada da dieta:

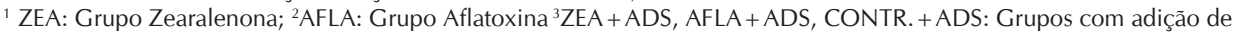
adsorvente

Para avaliação bioquímica foram coletadas amostras de soro, plasma (EDTA $10 \%)$ e plasma com adição de fluoreto de potássio (12\%) para determinação de glicose, triacilglicerol (TAG), colesterol, $\beta$ hidroxibutirato (BHBA), albumina, ureia, cálcio, fósforo, magnésio, $\gamma$-glutamil transferase (GGT) e aspartato aminotransferase (AST). As amostras de sangue foram centrifugadas à 3000 RPM, por 15 minutos, e conservadas resfriadas ou congeladas, de acordo com a análise a ser realizada. As análises bioquímicas foram feitas por meio de fotocolorimetria, utilizando espectrofotômetro de luz visível $\left(\right.$ FEMTO $435^{\circ}$ ).

A avaliação dos níveis séricos de albumina foi realizada em amostras de plasma resfriado, pelo método do verde de bromocresol (Albumina - Labtest Diagnóstica S.A., Brasil). Os níveis de glicose e ureia foram determinados pelos métodos da glicose oxidase (Glicose PAP Liquiform - Labtest Diagnóstica S.A., Brasil) e urease (Ureia CE - Labtest Diagnóstica S.A., Brasil), respectivamente, utilizando plasma resfriado com antiglicolítico. A avaliação dos níveis de TAG foi realizada nas amostras de soro resfriado, pelo método enzimáticoTrinder (Triglicérides Liquiform - Labtest Diagnóstica S.A., Brasil). O colesterol foi avaliado em amostras de soro congelado, utilizando o método de colesterol esterase oxidase (Colesterol Liquiform - Labtest Diagnóstica S.A., Brasil). A determinação dos níveis séricos das enzimas AST (AST/ GOT Liquiform - Labtest Diagnóstica S.A., Brasil) e GGT (Gama GT Liquiform - Labtest Diagnóstica S.A., Brasil) foi realizada em amostras de soro resfriado, utilizando os métodos cinético-UV e de Szasz modificado, respectivamente. As análises de cálcio total (Cálcio Liquiform - Labtest Diagnóstica S.A., Brasil), fósforo (Fósforo - Labtest Diagnóstica S.A., Brasil) e magnésio (Magnésio - Labtest Diagnóstica S.A., Brasil) também foram realizadas em amostras de soro resfriado. Para a determinação dos níveis séricos de BHBA (Ranbut - Randox Laboratories Ltd.) foi utilizada a metodologia cinética enzimática, em amostras de soro congelado.

Os dados obtidos deste experimento foram analisados no programa estatístico $\mathrm{SAS}^{7}$. As médias foram analisadas por meio do método MIXED MODELS, considerando o animal, o grupo e as coletas como efeitos fixos, e tendo níveis séricos de glicose, TAG, colesterol, BHBA, albumina, ureia, cálcio, fósforo, magnésio, GGT eAST como variáveis dependentes. A comparação de médias foi feita pelo teste Tukey HSD, sendo considerados significativos valores de $\mathrm{P}<0,05$.

\section{Resultados e Discussão}

Nas tabelas 2 e 3 estão demonstrados os níveis séricos dos marcadores energéticos, proteicos, minerais e enzimáticos avaliados neste experimento. 
Tabela 2- Valores médios (Coeficiente de Variação - \%) do perfil metabólico energético e enzimático de borregas submetidas a dietas contendo aflatoxina ou zearalenona, com ou sem adição do glucomanano modificado

\begin{tabular}{ccccccc}
\hline Metabólito & ZEA $^{1}$ & ZEA + ADS $^{2}$ & AFLA $^{3}$ & AFLA + ADS $^{2}$ & CONTR. + ADS $^{2}$ & CONTROLE $^{\text {a }}$ \\
\hline Glicose (mg/dL) & $42,0(19,4)^{\mathrm{c}}$ & $46,1(18,3)^{\mathrm{bc}}$ & $46,7(15,7)^{\mathrm{b}}$ & $49,8(16,4)^{\mathrm{ab}}$ & $51,9(18,0)^{\mathrm{a}}$ & $49,9(19,2)^{\mathrm{ab}}$ \\
TAG (mg/dL) & $33,1(56,5)$ & $33,0(57,6)$ & $29,5(73,0)$ & $38,7(59,8)$ & $32,0(57,9)$ & $30,3(41,8)$ \\
Colesterol (mg/dL) & $69,4(23,6)$ & $64,2(92,4)$ & $65,2(21,5)$ & $60,7(23,7)$ & $54,3(29,4)$ & $63,9(16,8)$ \\
BHBA (mg/dL) & $10,6(19,2)$ & $12,7(48,8)$ & $9,2(16,6)$ & $12,3(19,3)$ & $10,5(21,4)$ & $13,1(36,6)$ \\
AST (UI/l) & $82,5(20,4)^{\mathrm{a}}$ & $68,2(18,5)^{\mathrm{b}}$ & $68,8(18,7)^{\mathrm{b}}$ & $68,8(18,1)^{\mathrm{b}}$ & $71,0(25,8)^{\mathrm{b}}$ & $73,2(17,3)^{\mathrm{b}}$ \\
GGT (UI/l) & $114,4(76,8)^{\mathrm{a}}$ & $83,5(69,3)^{\mathrm{ab}}$ & $61,2(55,7)^{\mathrm{b}}$ & $83,6(78,7)^{\mathrm{ab}}$ & $76,2(67,1)^{\mathrm{b}}$ & $77,3(74,4)^{\mathrm{b}}$ \\
\hline
\end{tabular}

Valores com letras diferentes na mesma linha diferem estatisticamente pelo teste de Tukey $\operatorname{HSD}(\mathrm{p}<0,05)$;

${ }^{1}$ ZEA: Grupo Zearalenona; ${ }^{2} \mathrm{ZEA}+$ ADS, AFLA + ADS, CONTR. + ADS: Grupos com adição de adsorvente; ${ }^{3} \mathrm{AFLA}$ : Grupo Aflatoxina

Tabela 3 - Valores médios (Coeficiente de Variação - \%) do perfil metabólico proteico e mineral de borregas submetidas a dietas contendo aflatoxina ou zearalenona, com ou sem adição do glucomanano modificado

\begin{tabular}{ccccccc}
\hline Metabólito & ZEA $^{1}$ & ZEA + ADS $^{2}$ & AFLA $^{3}$ & AFLA + ADS $^{2}$ & CONTR. + ADS $^{2}$ & CONTROLE $^{\text {a }}$ \\
\hline Ureia (mg/dL) & $51,4(19,3)^{\mathrm{a}}$ & $51,7(13,0)^{\mathrm{a}}$ & $47,0(19,7)^{\mathrm{ab}}$ & $46,3(17,3)^{\mathrm{b}}$ & $48,4(16,3)^{\mathrm{ab}}$ & $46,3(21,1)^{\mathrm{b}}$ \\
Albumina (g/dL) & $2,5(21,7)$ & $2,4(23,4)$ & $2,3(29,9)$ & $2,3(27,9)$ & $2,4(26,0)$ & $2,5(16,5)$ \\
Cálcio (mg/dL) & $9,4(30,5)$ & $8,4(26,0)$ & $8,8(31,5)$ & $8,4(30,6)$ & $8,4(35,1)$ & $8,8(25,8)$ \\
Fósforo (mg/dL) & $9,0(21,6)$ & $8,7(26,9)$ & $9,2(32,3)$ & $9,3(27,6)$ & $8,3(23,8)$ & $9,5(25,3)$ \\
Magnésio (mg/dL) & $2,6(27,6)$ & $2,5(24,7)$ & $2,4(29,9)$ & $2,6(27,9)$ & $2,5(19,9)$ & $2,3(27,5)$ \\
\hline
\end{tabular}

Valores com letras diferentes na mesma linha diferem estatisticamente pelo teste de Tukey $\mathrm{HSD}(\mathrm{p}<0,05)$

${ }^{1}$ ZEA: Grupo Zearalenona; ${ }^{2} \mathrm{ZEA}+$ + ADS, AFLA + ADS, CONTR. + ADS: Grupos com adição de adsorvente; ${ }^{3} \mathrm{AFLA}$ : Grupo Aflatoxina

A adição de zearalenona na dieta das fêmeas diminuiu os níveis séricos de glicose, em relação ao CONTROLE $(\mathrm{p}<0,05)$, o que pode sugerir que estes animais tiveram um maior gasto de energia em relação aos outros grupos. Isto se deve, provavelmente, ao efeito anabólico de metabólitos da zearalenona, como o zeranol e o $\alpha$-zearalenol ${ }^{4}$. Porém, a adição de adsorvente náo influenciou os níveis de glicose, não havendo diferença entre os grupos ZEA e ZEA + ADS.

Os níveis séricos de TAG não diferiram entre os grupos ( $p>0,05)$, o que indica que estes receberam dietas com os mesmos níveis de gordura 8 .

Os níveis de colesterol e $\mathrm{BHBA}$ não apresentaram diferença entre os grupos ( $p$ $>0,05)$. Também, os níveis de colesterol se mantiveram dentro dos valores fisiológicos $(52-76 \mathrm{mg} / \mathrm{dL})^{8}$, enquanto que os valores de BHBA foram superiores ao limite fisiológico nos grupos ZEA + ADS, AFLA + ADS e CONTROLE $(6-10 \mathrm{mg} / \mathrm{dL})^{8}$.
Nos grupos ZEA e ZEA + ADS os níveis de ureia foram superiores ao CONTROLE $(p<0,05)$, indicando um maior metabolismo proteico em animais submetidos a dieta contendo zearalenona, porém dentro dos padrôes fisiológicos (4-10 $\mathrm{mmol} / \mathrm{L}=24-60 \mathrm{mg} / \mathrm{dL})^{8}$.

A albumina sérica não foi influenciada pela adiçấo de micotoxinas ou adsorvente à dieta, o que se deve ao fato deste marcador proteico ser mais estável do que a ureia, respondendo somente a alteraçóes por longos períodos nos níveis de proteína da dieta $^{9}$, não havendo tempo suficiente neste experimento para este tipo de alteração. Porém, em relação aos valores fisiológicos de albumina $(2,4-3,0 \mathrm{~g} / \mathrm{dL})^{8}$ os grupos AFLA e AFLA + ADS apresentaram níveis reduzidos deste marcador, o que pode ser devido a lesão hepática causada por esta micotoxina e a consequente inibiçãoo na síntese de proteína ${ }^{8}$.

Os níveis séricos de cálcio, fósforo e magnésio não foram influenciados pela 
presença de micotoxinas ou do adsorvente na dieta, o que indica que a presença do glucomanano modificado não interferiu na absorção intestinal destes minerais. Ainda, todos os minerais analisados apresentaram valores dentro dos padróes fisiológicos (Cálcio: 7,4-13 mg/dL; Fósforo: 2,0-9,6 mg/ $\mathrm{dL}$; Magnésio: 1,8-3,0 mg/dL) ${ }^{10}$.

A zearalenona aumentou significativamente os níveis de AST e GGT em relação ao grupo CONTROLE $(\mathrm{p}<0,05)$, sugerindo que esta micotoxina causou algum grau de lesão hepática. Possivelmente, os prejuízos reprodutivos causados pela zearalenona podem estar relacionados, além da ação direta no perfil hormonal, a um efeito indireto, por meio da alteração da funçáo hepática. Até o momento não foram descritas alteraçóes metabólicas causadas por esta micotoxina, sendo um dado novo e uma importante avaliação em posteriores experimentos. Assim, seriam necessários novos estudos, com uma avaliação mais detalhada do efeito de diferentes doses de zeralenona na atividade hepática de ruminantes, para confirmar esta hipótese. Ainda, todos os grupos apresentaram valores fisiológicos de AST e níveis de GGT acima dos valores de referência para a espécie ovina (AST: 0-90UI/L; GGT: 20-52 UI/L) ${ }^{11}$.

A partir da diferença encontrada entre os grupos ZEA e ZEA+ADS, quanto aos valores de AST, pode-se concluir que o adsorvente glucomanano modificado foi eficaz em reduzir o efeito deletério desta micotoxina sobre a atividade hepática. $\mathrm{O}$ aumento nos níveis plasmáticos desta enzima é indicativo de lesão hepática aguda, embora também possa ser produzida em alterações musculares. Já a enzima GGT, apresenta elevação em seus níveis somente em alteraçóes crônicas ${ }^{8}$, não sendo encontrada diferença entre os grupos ZEA e ZEA + ADS provavelmente devido ao curto período experimental.

Assim, pode-se concluir que a concentração de aflatoxina utilizada neste experimento não foi suficiente para causar alterações em parâmetros metabólicos. Estes resultados diferem daqueles obtidos por
Suliman et al. ${ }^{12}$, em que uma contaminação com somente $0,75 \mathrm{mg} / \mathrm{kg}$ foi suficiente para causar alteraçôes bioquímicas, lesões hepáticas e óbitos. Isto se deve, provavelmente, ao fato da aflatoxina apresentar maior toxicidade quando proveniente de contaminaçóes naturais da dieta, em relação à sua forma purificada. Esta diferença parece ser causada pelo sinergismo existente entre a aflatoxina e outras micotoxinas ${ }^{13}$. Assim, seriam necessários outros estudos, avaliando diferentes doses de aflatoxina, bem como a sua interação com outras micotoxinas, para que possa ser determinada a sua atuaçáo sobre os parâmetros analisados, além de determinar de forma mais adequada o efeito do adsorvente glucomanano modificado sobre esta micotoxina. Outra possibilidade é a realização de um período experimental mais longo, permitindo que sejam avaliadas alteraçóes a longo prazo, as quais não foram expressas neste experimento.

Estes resultados também demonstram que a zearalenona causa um desequilíbrio metabólico em ovinos, devido possivelmente à agressáo hepática causada por esta micotoxina, caracterizada pelos altos níveis séricos de AST e ureia, bem como pelos níveis reduzidos de glicose observados nestas fêmeas. Ainda, para que seja elucidado o efeito da zearalenona sobre o metabolismo de ruminantes, independente de seus metabólitos, seria necessária a utilizaçáo de zearalenona pura, diferentemente do cultivo de Fusarium utilizado neste experimento.

Pela comparação entre os grupos CONTROLE e CONTROLE + ADS pôde-se verificar que a administraçáo do glucomanano modificado na dieta de ruminantes náo apresenta efeito deletério sobre a absorção de nutrientes, visto que não foram observadas diferenças estatísticas ( $p$ > 0,05) nos parâmetros analisados.

\section{Conclusões}

Quanto ao efeito do glucomanano modificado sobre as alteraçóes metabólicas causadas por estas micotoxinas, pode-se 
concluir que este adsorvente foi eficiente em reduzir a possível agressão hepática causada pela zearalenona, demonstrada pela diminuição nos níveis de AST. Ainda, a aflatoxina causou uma redução nos níveis de albumina, em relação aos valores fisiológicos de ovinos. Também, pôde-se observar que o adsorvente não teve efeito sobre os níveis séricos de minerais.

\section{Agradecimentos}

Os autores agradecem à Alltech e ao $\mathrm{CNPq}$ pelo financiamento desta pesquisa.

\title{
Metabolic parameters of ewes receiving diets containing a flatoxin and zearalenone with addition of modified glucomannan
}

\begin{abstract}
The micotoxicoses are causing great losses of production in ruminants, being aflatoxin (AFLA) and zearalenone (ZEA) the major mycotoxins found in foods preserved. These mycotoxins have effect on the metabolism animal through the anabolic action of metabolites of the ZEA, and the liver injury caused by AFLA. The purpose of this study was to determine the influence of the sorbent modified glucomannan on metabolic parameters of sheep submitted to diets containing AFLA and ZEA. For this, 34 females were used and they were divided into 6 groups (ZEA; ZEA + ADS; AFLA; AFLA + ADS; CONTROL + ADS; CONTROL), receiving $1.0 \mathrm{mg} / \mathrm{kg}$ of ZEA, $1.5 \mathrm{mg} / \mathrm{kg}$ of AFLA and/or $2 \mathrm{~kg} / \mathrm{ton}$ of sorbent. The ZEA decreased serum levels of glucose, for the CONTROL $(p<0.05)$, however, the sorbent not influence the levels of glucose, with no difference between groups ZEA and ZEA + ADS. The ZEA increased levels of AST and GGT compared to group CONTROL $(p<0.05)$, whereas the levels of AST were higher in the group ZEA $(\mathrm{p}<0.05)$ when compared to the group ZEA+ADS. Still, the aflatoxin caused a reduction in the levels of albumin, for physiological values of sheep. Thus, from these results it was concluded that ZEA caused metabolic alterations in sheep, and the modified glucomannan was effective in reducing the possible liver aggression caused by this mycotoxin, shown by the decrease in the levels of AST.
\end{abstract}

\section{Referências}

1 JOUANY, J-P.; DIAZ, D. E. Effects of mycotoxins in ruminants. In: DIAZ, D. E. The mycotoxin blue book. Nottingham: University Press, 2005. p. 295-321.

2 EDRINGTON, T. S.; HARVEY, R. B.; KUBENA, L. F. Effect of aflatoxin in growing lambs fed ruminally degradable or escape protein sources. Journal of Animal Science, v. 72, n. 5, p. 1274-1281, 1994.

3 D'MELLO, J. P. F.; PLACINTA, C. M.; MACDONALD, A. M. C. Fusarium mycotoxins: a review of global implications for animal health, welfare and productivity. Animal Feed Science and Technology, v. 80, n. 3/4, p. 183-205, 1999.

4 KENNEDY, D. G.; HEWITT, S. A.; MCEVOY, J. D.; CANNAVAN, A.; BLANCHFLOWER, W. J.; ELLIOT, C.
Keywords:

Aflatoxin.

Zearalenone.

Modified glucomannan. Metabolism.

Sheep.
T. Zeranol is formed from Fusarium sp. toxins in cattle in vivo. Food Additives and Contaminants, v. 15, n. 4 , p. 393-400, 1998.

5 ARAVIND, K. L.; PATEL, V. S.; DEVEGOWDA, G.; UMAKANTHA, B.; GANPULES, S. P. Efficacy of esterified glucomannan to counteract mycotoxicosis in naturally contaminated feed on performance and serum biochemical and hematological parameters in broilers. Poultry Science, v. 82, n. 4, p. 571-576, 2003.

6 RUSSEL, A. J. F.; DONEY, J. M.; GUNN, R. G Subjective assessment of body fat in sheep. Journal Agricultural Science, v. 72, n. 3, p. 451-454, 1969.

7 STATISTICAL ANALYSIS SYSTEM (SAS). Principles and procedure of statistics. 2. ed. Cary: Mc Graw-Hill Inc., 1986. 
8 GONZÁLEZ, F. H. D.; SILVA, S. C. Introdução à bioquímica clínica veterinária. Porto Alegre: UFRGS, 2003. 198 p.

9 CALDEIRA, R. M.; BELO, A. T.; SANTOS, C. C.; VAZQUES, M. I.; PORTUGAL, A. V. The effect of long-term feed restriction and over-nutrition on body condition score, blood metabolites and hormonal profiles in ewes. Small Ruminant Research, v. 68, n. 3, p. 242-255, 2007.

10 GONZÁLEZ, F. H. D. Indicadores sanguíneos do metabolismo mineral em ruminantes. In: DIAZ GONZÁLEZ, F. H.; BARCELLOS, J. O.; RIBEIRO, L. A. O. (Ed.). Perfil metabólico em ruminantes: seu uso em nutrição e doenças nutricionais. Porto Alegre: UFRGS, 2000. p. 31-52.
11 CONTRERAS, P.; WITTWER, F.; BÖHMWALD, H. Uso dos perfis metabólicos no monitoramento nutricional dos ovinos. In: DIAZ GONZÁLEZ, F. H.; BARCELLOS, J. O.; RIBEIRO, L. A. O. (Ed.). Perfil metabólico em ruminantes: seu uso em nutrição e doenças nutricionais. Porto Alegre: UFRGS, 2000. p. 75-88.

12 SULIMAN, H. B.; MOHAMED, A. F.; AWADELSIED, N. A.; SHOMMEIN, A. M. Acute mycotoxicosis in sheep: Field cases. Veterinary and Human Toxicology, v. 29, n. 3, p. 241-243, 1987.

13 APPLEBAUM, R. S.; BRACKETT, R. E.; WISEMAN, D. W.; MARTH, E. H. Response of dairy cows to dietary aflatoxin: Feed intake and yield, toxin content and quality of milk of cows treated with pure and impure aflatoxin. Journal of Dairy Science, v. 65, n. 8, p. 1503-1508, 1982. 